\title{
Pre-charging of module capacitors of MMC when the module switches are driven by a source derived from the module capacitor
}

\author{
SHAMKANT D JOSHI*, M C CHANDORKAR and A SHUKLA \\ Department of Electrical Engineering, Indian Institute of Technology Bombay, Mumbai 400076, India \\ e-mail: shamkant@ee.iitb.ac.in; sj20124@gmail.com
}

MS received 31 March 2016; revised 8 September 2016; accepted 3 October 2016

\begin{abstract}
A modular multilevel converter (MMC) is one of the latest multilevel converters used for high and medium-voltage power conversion. It is based on cascade connection of multiple identical modules using IGBTs as switching devices. Module switches of MMC are preferably driven by a source derived from the module capacitor. In each MMC module, the control circuit, consisting of gate drivers, is powered from a dc supply derived from the local capacitor. The module capacitors need to be pre-charged, to power the control circuit. The problem faced while doing so experimentally for MMC with two modules per arm and a solution have been reported earlier. If a fly-back converter is used to generate the power supply for driving the control circuit, the module capacitor voltages become unstable during uncontrolled pre-charging. It has been reported earlier that the reason for this is approximately constant power load on the module capacitor. This work provides theoretical understanding of the problem and shows by analysis that the power supply can be made stable if the load on the module capacitor is made a positive resistance load. As the complexity of MMC with more than two modules per arm is higher, the phenomenon is studied by simulation for MMC with four modules per arm. It shows that when a fly-back converter is used for generating the power supply, similar instability occurs in MMC with four modules per arm. It shows that when the module capacitor is made to have a load with positive resistance characteristics, the module capacitor voltages and consequently the power supplies stabilize even for MMC with four modules per arm. It further shows that even if the load on the module capacitor is negative resistance type, when fly-back converter is used to generate the module power supply, by switching devices in those modules where power supply becomes available first, followed by sorting algorithm, stable power supplies can be developed on all the modules and the capacitors can be fully charged to the desired voltage.
\end{abstract}

Keywords. Capacitor pre-charging for cascaded modules; MMC modules using fly-back converter; MMC modules using forward converter; power supply from local module capacitor.

\section{Introduction}

The paper [1] presented a new multilevel converter topology, termed as modular multi-level converter (MMC), suitable for very-high-voltage applications, especially power generation and transmission. The fundamental concept and the applied control scheme was introduced. The MMC has a modular arm structure with cascaded connection of multiple bidirectional chopper cells with floating capacitors. The greatest advantage of MMC is the modular construction. The modules used in the MMC can be either half bridge or full bridge cell type. The half-bridge-type cell is shown in figure 1. It consists of two insulated gate bipolar transistor (IGBT) switches $S_{1}$ and $S_{2}$ with anti-parallel diodes. A capacitor connected as shown in the figure is kept charged. The module has only two terminals. When the top switch $S_{1}$ or the anti-parallel diode $D_{1}$ conducts, the capacitor voltage $V_{C}$ is impressed across the

*For correspondence input terminals, resulting in $V_{X}=V_{C}$. When the bottom switch $S_{2}$ or the anti-parallel diode $D_{2}$ conducts, the capacitor is bypassed and $V_{X}=0$. Figure 2 shows a three-phase MMC. It consists of three legs to share a common DC link voltage. Each of these legs has two arms, and each arm has a string of series-connected modules and an inductor. Half-bridge modules can produce two voltage levels $\left(0\right.$ or $\left.V_{C}\right)$ across its terminal. Some of the popular modulation and control strategies for this converter are discussed in [2-6].

A large number of MMC modules, connected in series are employed for high-power converters. If the power for IGBT drivers is supplied externally, isolating transformers have to cope with high isolation requirements and electromagnetic-compatibility (EMC) issues as described in [7]. Therefore, each gate driver is powered from a dc supply derived from the local capacitor. In [7] auxiliary voltage source with relatively lower voltage level is used to charge the module capacitors initially. The structure of the MMC module is explained in [8]. A fly-back converter is a natural 


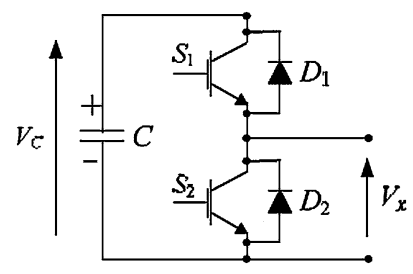

Figure 1. Half-bridge cell with capacitor.

choice of a dc power supply derived from the local capacitor because of its low component count and higher efficiency. It was found experimentally in $[9,10]$ that, when a fly-back converter is used to derive the power supply from the module capacitor, in the pre-charging state, the module capacitor voltage and, consequently, the power supplies do not stabilize.

Single-phase MMC circuit hardware was developed and tested as reported in $[9,10]$. The circuit diagram is shown in figure 3. It was observed that the capacitor voltages $V_{C 1}$ $-V_{C 4}$ across the four module capacitors fluctuate when the power supply is derived using a regulated fly-back converter. It was further explained that this was due to the approximately constant power load on the module capacitor by the SMPS. It was also shown experimentally that the module capacitor voltages can be made stable using a forward converter followed by a linear regulator [9].
In the present work, the aforementioned experimental observations when (a) power supply is derived using a flyback converter and (b) power supply is derived using a forward converter in open loop followed by linear regulator have been analysed theoretically. Pre-charging of MMC with four modules per arm is simulated for the cases where the module power supply is developed using a (a) fly-back converter and (b) forward converter in open loop followed by linear regulator. The fly-back converter load on module capacitor is modelled as a constant-power load and the phenomenon of instability is verified by simulation. It is shown that if the load is made linear, the capacitor voltages in pre-charging stage stabilize. It is further shown that the capacitor voltages can be stabilized in controlled precharging state, even with constant-power load, by switching the devices for which power supply is ready, followed by sorting algorithm, to balance the capacitor voltage.

\section{Analysis of the stability issue when the power supply is derived using a regulated fly-back converter}

To analyse the stability issue, let us examine the load presented by a regulated fly-back converter to the module capacitor of an MMC module. Consider a typical MMC module with the power supply $V_{01}$ derived from the module

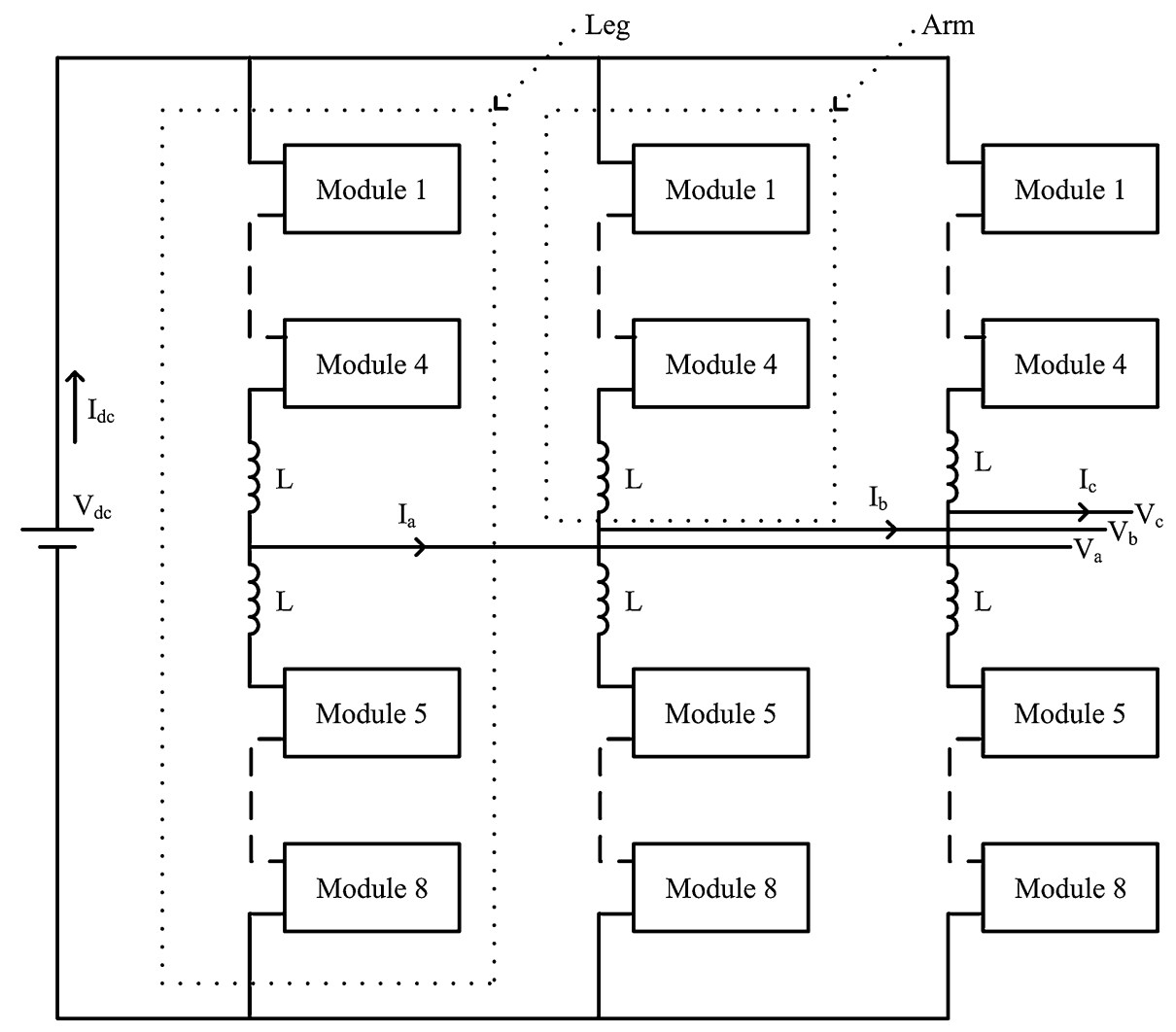

Figure 2. Three-phase modular multilevel converter. 


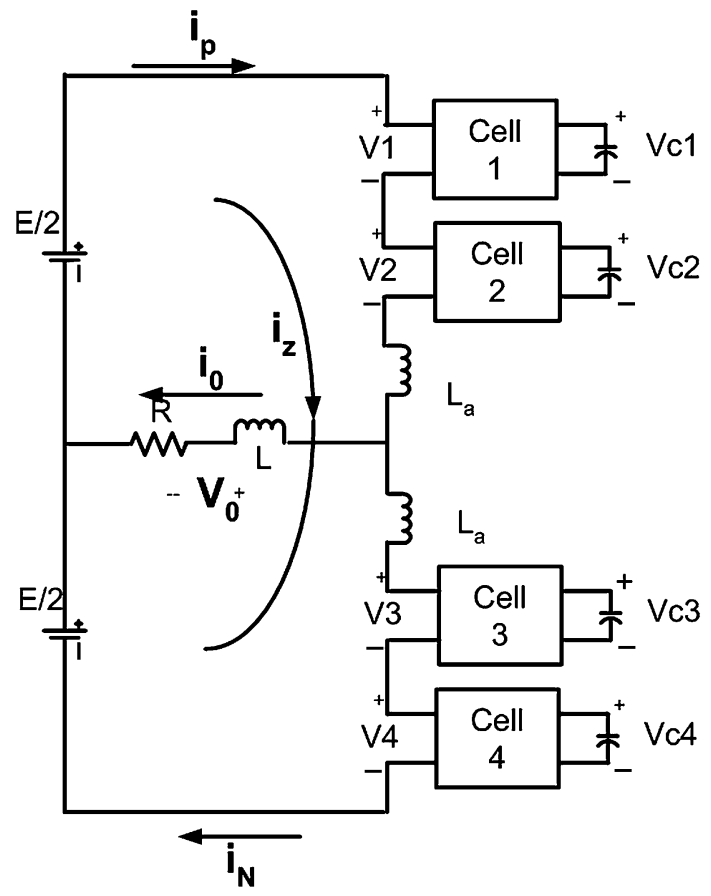

Figure 3. single-phase MMC circuit with four modules per leg.

capacitor voltage $V_{C 1}$ by a regulated fly-back converter as shown in figure 4 . When the voltage $V_{01}$ is regulated and there is a small increase in the capacitor voltage $V_{C 1}$, the fly-back converter should reduce the duty cycle to maintain the output voltage $V_{01}$. Assuming that the efficiency of the fly-back converter remains constant for a small change in input voltage, the average current $I_{C 1}$ reduces. Therefore for a given arm current the charging current $I_{\mathrm{ch}}$ increases, thus increasing the capacitor voltage further. The capacitor voltage has a tendency to be unstable. Increase in capacitor voltage decreases the current flowing out of the positive terminal. This is a negative resistance characteristic.

\subsection{The load to the module capacitor and stability with one MMC module}

Consider the stability of a system with only one capacitor with negative resistance load.

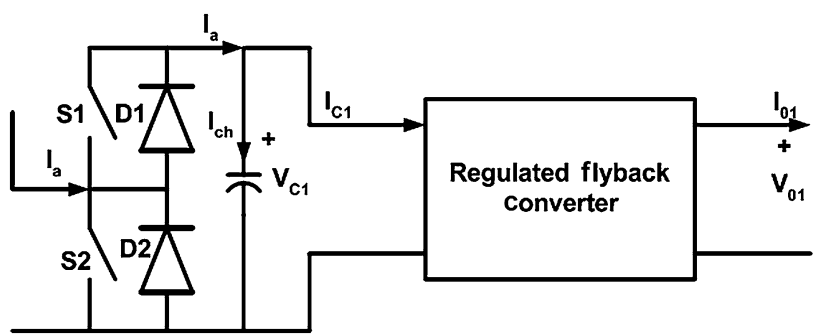

Figure 4. Load presented to module capacitor by a regulated flyback converter.
Figure 5 shows a single-module capacitor charging through a resistor $R$ and an inductor $L$. The resistor $R$ is included to limit the initial current. The load across the module capacitor is represented by a negative resistance $-R_{1}$, where $R_{1}$ is a positive real number representing the absolute value of the resistance at the operating point. As discussed earlier, since the load on the capacitor can be considered as approximately a constant-power load when fly-back converter is used, the load has a negative resistance characteristic at any operating point. The negative resistance is represented by $-R_{1}$. If $V_{c}$ and $E$ are incremental voltages, the open-loop transfer function considering $V_{c}$ as output voltage and $E$ as input voltage can be written as follows:

$$
\frac{V_{c}}{E}=\frac{R_{1}}{s^{2} L C R_{1}+s\left(C R R_{1}-L\right)+\left(R_{1}-R\right)} .
$$

Equation (1) shows that the necessary and sufficient conditions for $V_{c}$ to be stable are

$$
\left(R_{1}-R\right)>0
$$

$$
\left(C R R_{1}-L\right)>0 \text {. }
$$

The resistor $R$ in figure 5 is selected to limit the initial charging current and its value is taken as $50 \Omega$. After precharging, it is reduced to $5 \Omega$. The resistance $R_{1}$ is evaluated based on constant-power load for the IGBT driver circuit equal to $2.5 \mathrm{~W}$. It varies with the module capacitor voltage. The graph for the same is given in figure 6. From the graph, $R_{1}$ is always greater than $R$. Hence Eq. (2) is valid. With $C=1 \mathrm{mF}$ and $L=3 \mathrm{mH}$, Eq. (3) is valid. Hence the voltage $V_{c}$ in figure 5 is stable. The theory corroborates with the experimental findings in [9].

\subsection{Stability with two MMC modules in series}

Consider two MMC modules connected in series with a power supply derived by a regulated fly-back converter as shown in figure 7 . Since all the switches S1-S4 are off, the

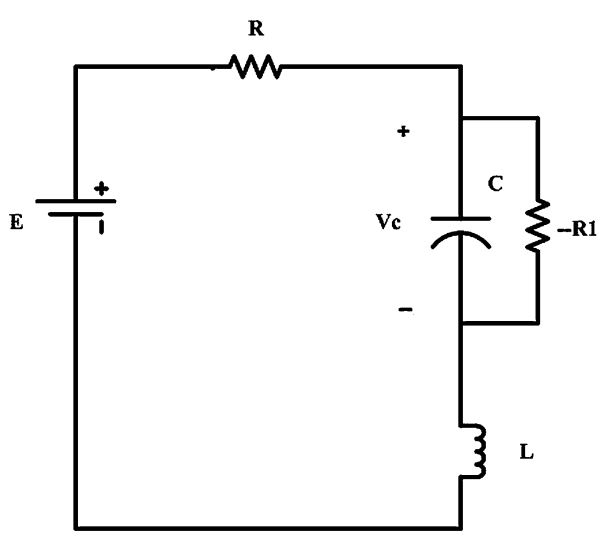

Figure 5. Capacitor charging of a single module. 


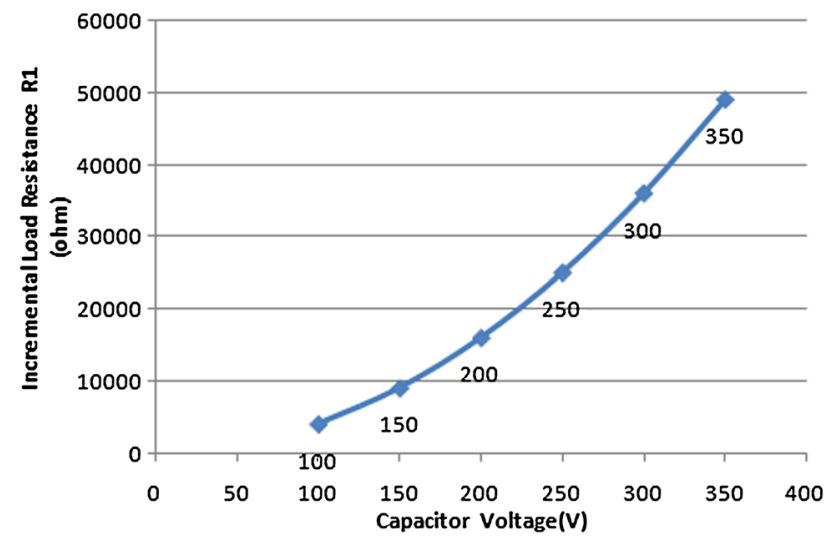

Figure 6. Incremental load resistance variation with capacitor voltage.

capacitors are charged by the same current $I_{a}$ and discharged by currents $I_{c 1}$ and $I_{c 2}$. Consider a small increase in voltage $V_{c 1} . I_{c 1}$ should reduce for the given load. Since the discharging current reduces, the voltage $V_{c 1}$ will increase further and will go on increasing. Since both the capacitors are being supplied by the same source, increase in one capacitor voltage should result in decrease in the other capacitor voltage. The two voltages diverge and will make the system unstable. With more number of modules in series the situation is more complex. The load on the capacitor has a negative resistance characteristic, since increase in voltage results in decrease in discharge current.

If load on the capacitors is such that increase in voltage results in increase in discharge current, then there is a stabilizing effect. This can be achieved using a forward converter in open loop at fixed-duty cycle, followed by a linear regulator. It was observed experimentally in [9] that the capacitor voltage stabilizes for two modules in series.

Figure 8 shows capacitors of two modules charging through a resistor $R$ and an inductor $L$. The resistor $\mathrm{R}$ is included to limit the initial current. The load across the

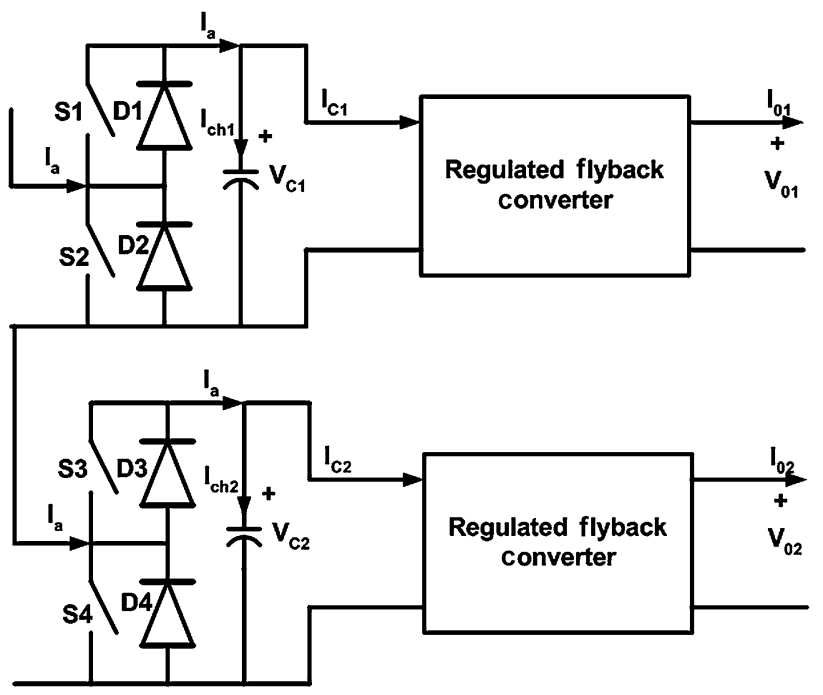

Figure 7. Stability with two MMC modules in series.

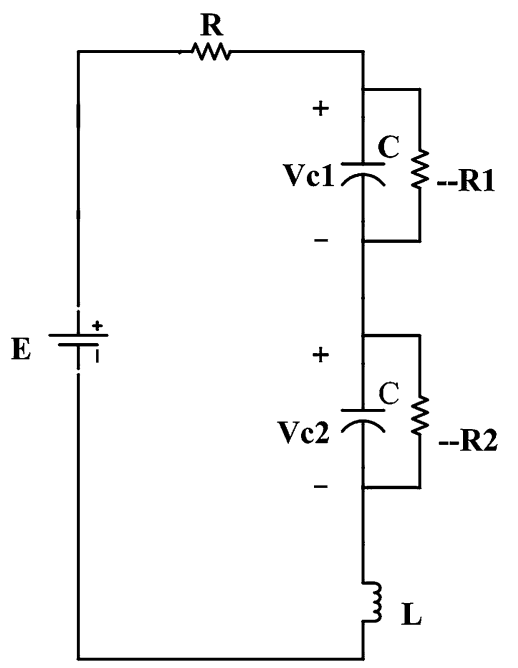

Figure 8. Capacitor charging of two modules.

module capacitor is represented by negative-resistances $-R_{1}$ and $-R_{2}$ at the operating point. $R_{1}$ and $R_{2}$ are positive real numbers. As discussed earlier, since the load on the capacitor can be considered as approximately a constantpower load when a regulated fly-back converter is used, the load has a negative-resistance characteristic at any operating point. If $V_{c 1}, V_{c 2}$ and $E$ are incremental voltages, the open-loop transfer function considering $V_{c 1}$ as output voltage and $E$ as input voltage can be written as Eq. (4).

From Eq. (4) and figure 6, since $R_{1}$ and $R_{2}$ are far greater than $R$, the denominator has the first and the last terms with opposite sign. Hence the system is unstable as per Routh's Stability Criterion. The theory corroborates with the experimental findings in [9].

It is to be noted that the instability is not due to $L C$ oscillation. If the value of $L$ in Eq. (4) is set to zero and Eq. (5) is obtained, co-efficients of at least two terms in the denominator have opposite sign and the system is unstable as per Routh's Stability Criterion.

From simulation results as indicated in figure $9, V_{c 1}$ and $V_{c 2}$ are unstable as shown by the two diverging voltages. If there are two capacitors in series with negative load resistance on each, the capacitor voltages are seen to be unstable as indicated by theory, simulation and experiment [9].

\section{Analysis of the stability issue when the power supply is derived using a forward converter followed by linear regulator}

\subsection{Stability of the module capacitor voltages when the load on the module capacitor has positive resistance characteristics}

If the load resistances across the two module capacitors are positive, the transfer function of the incremental voltage across one of the capacitors is given by Eq. (6). 


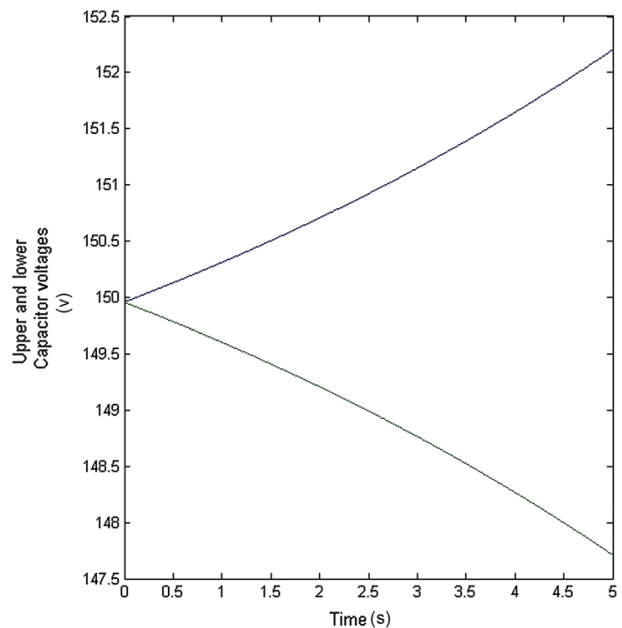

Figure 9. Simulation of capacitor charging of two modules.

From Eq. (6) it is seen that all the coefficients in the numerator and the denominator of the transfer function are positive. All the poles and zeros are in the left half plane. Applying Routh's Stability Criterion, all the coefficients being positive is only a necessary condition for stability in the present case because the denominator is of third order. It is shown in detail in Appendix that the above system is

\subsection{MMC module powered from an open-loop forward converter followed by a linear regulator}

Figure 10 shows a block diagram for a forward converter followed by a linear regulator. The forward converter is operated in open loop at a constant-duty cycle and converts the capacitor dc voltage $V_{c}$ in the range of 100-300 V to the dc voltage $V_{i}$ in the range of 20-60 V. $V_{i}$ is then applied to the input of a linear regulator, which regulates the voltage $V_{o}$ at its output to a voltage level of 14-15 V. The linear regulator in figure 10 was realized in the experimental setup in [9] by a TL783 ("TL783 High Voltage Adjustable Regulator" Application note, Texas Instruments, 2008). A schematic of the same is shown in figure 11.

The voltage $V_{o}$ in figures 10 and 11 was used as the power supply for the IGBT drivers and module capacitor voltage sensor in [9]. The output voltage $V_{o}$ is given by Eq. (8). The first term in Eq. (8) does not vary with the input voltage. However, the variable $I_{a d j}$ in the second term increases with input voltage $V_{i}$. As $V_{o}$ increases the load current increases. The capacitor voltage $V_{c}$ is directly proportional to the voltage $V_{i}$ and the current drawn from the module capacitor increases with increase in capacitor voltage $V_{c}$. Hence the load as seen by the module capacitor has positive resistance characteristics. A Detailed derivation showing that the present system is stable is given in Appendix.

$$
\frac{V_{c 1}}{E}=\frac{\left(R_{1} R_{2} C s-R_{1}\right)}{s^{3} R_{1} R_{2} L C^{2}+s^{2}\left(R R_{1} R_{2} C^{2}-L C R_{1}-L C R_{2}\right)+s\left(L-C R R_{1}-C R R_{2}\right)+\left(R-R_{1}-R_{2}\right)}
$$

stable.

Since the initial pre-charging is uncontrolled, series inductance $L$ is not required initially and can be bypassed. If the value of $L$ in Eq. (4) is set to zero, Eq. (6) reduces to Eq. (7) . All the co-efficients in the denominator, that is a second-order equation, are positive. Hence the system is stable as per Routh's Stability Criterion.

$$
\frac{V_{c 1}}{E}=\frac{\left(R_{1} R_{2} C s-R_{1}\right)}{s^{2}\left(R R_{1} R_{2} C^{2}\right)-s C R\left(R_{1}+R_{2}\right)+\left(R-R_{1}-R_{2}\right)}
$$

$$
\frac{V_{c 1}}{E}=\frac{\left(R_{1} R_{2} C s+R_{1}\right)}{s^{3} R_{1} R_{2} L C^{2}+s^{2}\left(R R_{1} R_{2} C^{2}+L C R_{1}+L C R_{2}\right)+s\left(L+C R R_{1}+C R R_{2}\right)+\left(R+R_{1}+R_{2}\right)}
$$

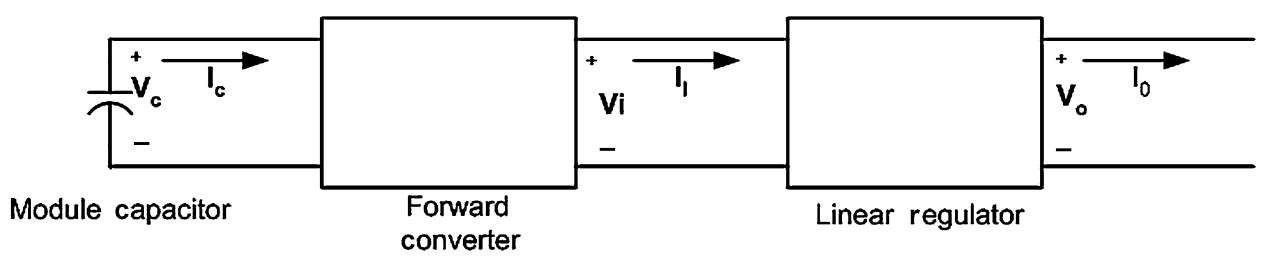

Figure 10. Forward converter followed by a linear regulator. 


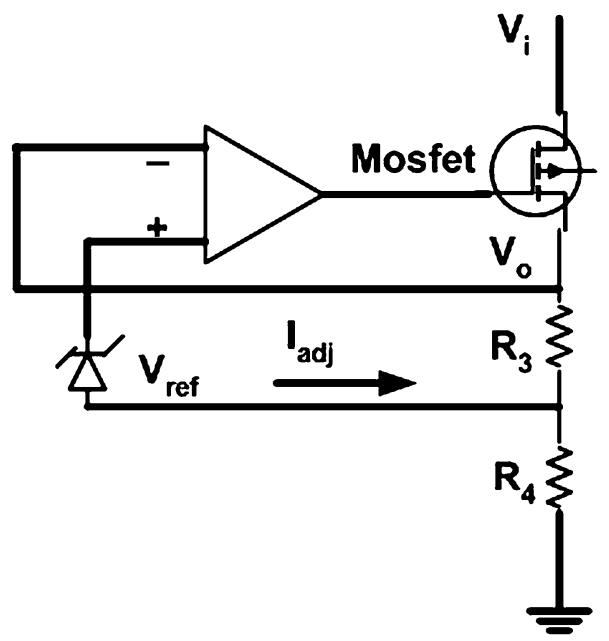

Figure 11. Linear regulator.

$$
\frac{V_{c 1}}{E}=\frac{\left(R_{1} R_{2} C s+R_{1}\right)}{s^{2}\left(R R_{1} R_{2} C^{2}\right)+s\left(C R R_{1}+C R R_{2}\right)+\left(R+R_{1}+R_{2}\right)}
$$

$$
V_{o}=V_{r e f}\left(1+\frac{R_{4}}{R_{3}}\right)+I_{a d j} R_{4}
$$

\section{Pre-charging of three phase MMC with four modules per arm}

From the analysis of pre-charging as described in section 2, analysis of the pre-charging process for MMC with more number of modules per arm would be quite complex as transfer functions of order higher than three will be involved. Hence, insight into the pre-charging of threephase MMC with four modules per arm is obtained in this work based on simulation.

\subsection{Uncontrolled pre-charging}

Since the power for driving the IGBT switches, as shown in figure 1, is derived from the module capacitor, no control is possible unless there is sufficient charge on the capacitor. Hence initial charging of the capacitor must be uncontrolled. It is achieved by connecting a resistor $R_{c}$ in series with each arm, as shown in figure 12, to limit the initial current. Since no control is available, switches S1 and S2 of all the modules are off and all the capacitors charge through diode D1 of the modules as seen in figures 1 and 12. All the modules M1 - M8 of all the three phases A, B and C in figure 12 are identical. Each capacitor gets charged to $V d c / 2 n$ for ' $n$ ' number of modules per arm. Power supply for each module is now derived from the charged capacitor.

A three-phase MMC, with four modules per arm, was simulated for pre-charging performance for the cases where the power supply of the module was derived from the module capacitor itself. In the previous section, it is shown that the load as seen by the module capacitor, when a flyback converter is used, is of approximately constant power type that is also a negative-resistance-type load. Hence the effect of fly-back converter is modelled as a constant-power load having a negative resistance characteristic. Similarly the effect of forward converter followed by a linear regulator is modelled as a positive resistance load. With four modules per arm as shown in figure 12 , and $V_{\mathrm{dc}}=600 \mathrm{~V}$, after uncontrolled pre-charging is complete, each capacitor should have approximately $75 \mathrm{~V}$. For obtaining a regulated output voltage of $15 \mathrm{~V}$ required for gate pulses, it was assumed that capacitor voltage should reach at least $55 \mathrm{~V}$. For capacitor voltage higher than $55 \mathrm{~V}$ the output voltage is regulated to $15 \mathrm{~V}$. When the output voltage reduces, it was assumed that $15 \mathrm{~V}$ regulated output will be obtained till it goes to $40 \mathrm{~V}$ and output will be zero below $40 \mathrm{~V}$. The hysteresis is necessary to avoid unnecessary oscillations at the changeover points. At $0.3 \mathrm{~s}$, the series resistors in the arm used for limiting the initial current are bypassed, allowing the capacitors to charge fully.

\subsection{Selection of current limiting resistance during uncontrolled pre-charging}

The currents in the circuit during uncontrolled precharging are completely decided by circuit parameters. The equivalent resistor inserted in each phase should

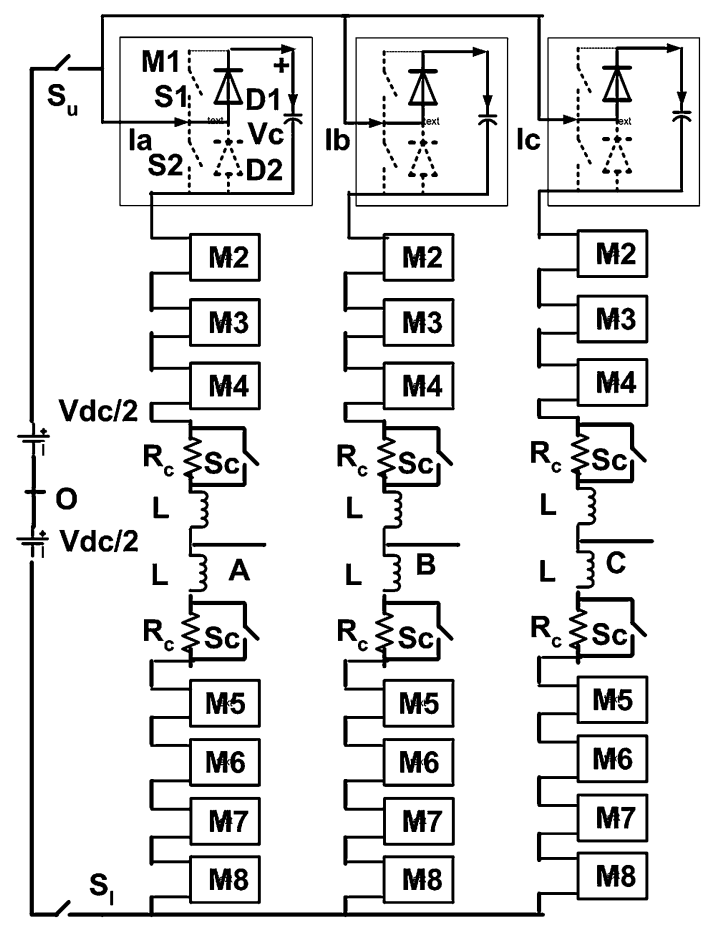

Figure 12. Uncontrolled pre-charging of three-phase modular multilevel converter. 
limit the initial current, as initially the module capacitors are in a completely discharged state. As mentioned in section 2, during uncontrolled pre-charging, the arm inductor is not required and can be bypassed. In that case the resistance is decided by the maximum current surge that the IGBTs used can allow. With dc bus voltage equal to $600 \mathrm{~V}$, the surge current can be limited to $20 \mathrm{~A}$ by inserting $R_{c}=15 \Omega$. However, additional switches are required to bypass the inductors. In the present case, arm inductors are not bypassed. The arm inductors and capacitors can result in oscillations, resulting in excessive currents. Hence the system is designed for overdamped operation. In figure 12, if the module capacitor value is ' $c$ ', with eight modules per leg and two arm inductors and two current-limiting resistors per leg, the value of resistance $R_{c}$ is given by

$$
R_{c} \geqslant 4 \sqrt{\left(\frac{L}{c}\right)} .
$$

Choice of $L=3 \mathrm{mH}$ and $c=1 \mathrm{mF}$ results in $R_{c} \geqslant 6.93 \Omega$. $R_{c}=15 \Omega$ was selected for the application. $R_{c}=15 \Omega$ can be used even if arm inductors are bypassed during uncontrolled pre-charging.

\subsection{Modeling the load on the capacitor due to regulated fly-back converter}

When the regulated output is at $0 \mathrm{~V}$, a small constant load is assumed across the capacitor. When the regulator output voltage is $15 \mathrm{~V}$, referring to figure 7 , the capacitor is assumed to have constant-power load, neglecting losses in the regulator. The load on the capacitor is almost constant because the main load on the power supply is the IGBT driver load of the two IGBTs in the module and the gate drives to the two IGBTs are complementary. Constantpower load has a negative resistance characteristic because when the voltage across the capacitor increases, the current drawn from the capacitor will decrease. figure 13 shows capacitor voltages of four modules in one arm and the arm current, as obtained by simulation. Ideally each module capacitor should get charged to $75 \mathrm{~V}$. Simulation results show that module 1 capacitor voltage crosses $200 \mathrm{~V}$, module 2 capacitor voltage crosses $100 \mathrm{~V}$ and then starts reducing, whereas module 3 and 4 voltages vary between 40 and $55 \mathrm{~V}$, which correspond to hysteresis points, where the load to the capacitor switches. The voltage variations of module 3 and module 4 capacitors are similar to those obtained experimentally in [9]. The modules, where capacitor voltage goes below $40 \mathrm{~V}$, will not have regulated
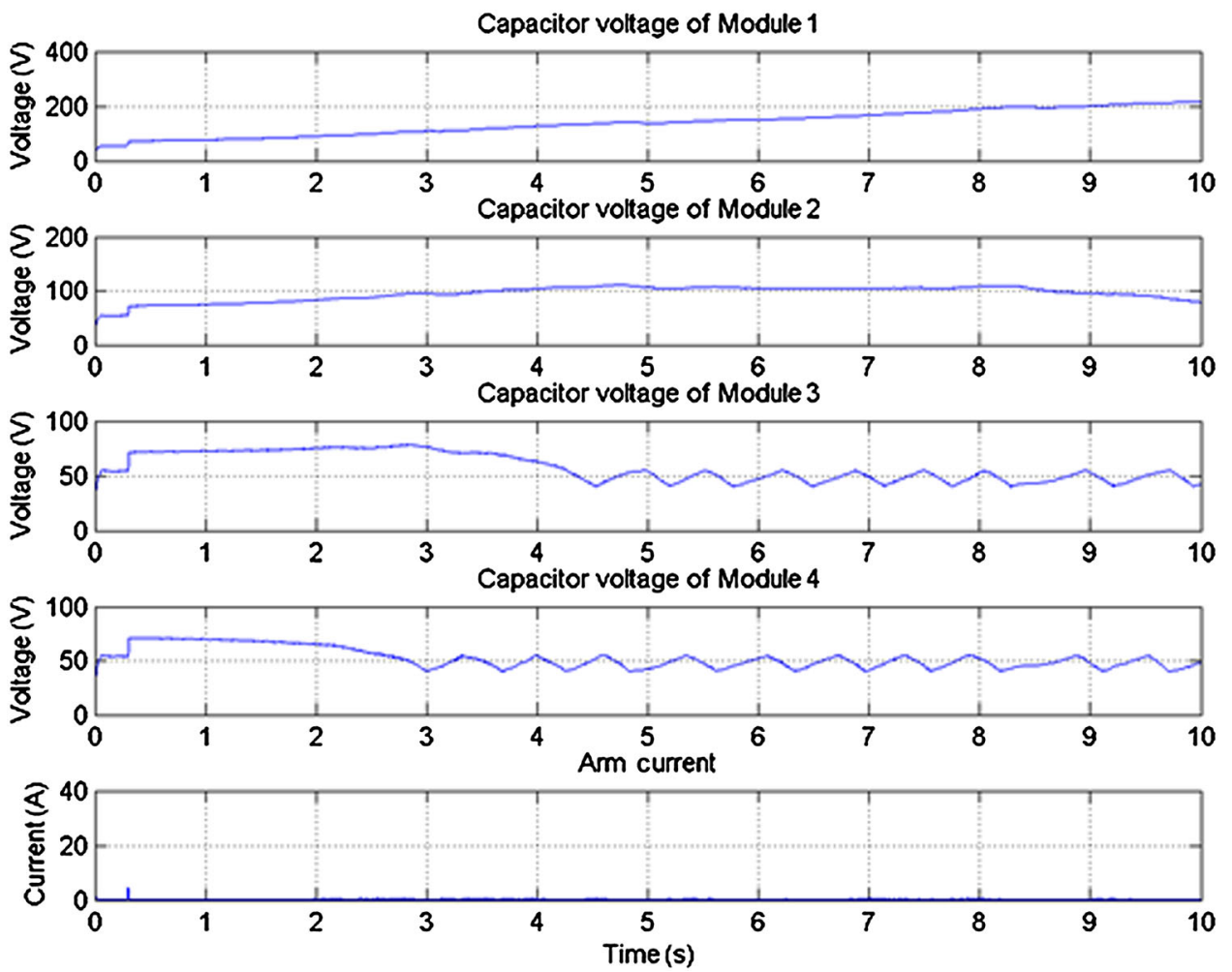

Figure 13. Uncontrolled pre-charging of capacitors when fly-back power supply is used. 
output voltage, and therefore they are not ready for switching IGBT devices. The conclusion is that some of the capacitor voltages do not reach desired values and the corresponding modules do not have regulated output voltage of $15 \mathrm{~V}$, whereas some capacitors will have higher than the minimum desired value at uncontrolled pre-charging and hence the corresponding power supply will be regulated to $15 \mathrm{~V}$. The IGBTs of modules having $15 \mathrm{~V}$ regulated supply can be switched and IGBTs of modules not having $15 \mathrm{~V}$ supply cannot be switched.

\subsection{Modeling the load on the module capacitor due to forward converter followed by linear regulator}

When the regulated output is at $0 \mathrm{~V}$, a small constant load is assumed across the capacitor. When the regulator output voltage is $15 \mathrm{~V}$, referring to figure 7 , the load on the capacitor is assumed to have an equation representing positive resistance characteristic:

$$
I_{c i}=m V_{c i}+c
$$

where ' $i$ ' varies from 1 to 8 for the eight modules in one leg; $m$ and $c$ are positive constants.

Figure 14 shows the capacitor voltages of four modules in one arm and the arm current. Ideally each module capacitor should get charged to $75 \mathrm{~V}$. Simulation results show that all the four modules in an arm reach $75 \mathrm{~V}$ and are stable at that value. Hence the regulator output voltages of all modules are at $15 \mathrm{~V}$ as desired. All the power supplies are ready for switching.

\section{Controlled pre-charging of three-phase MMC with four modules per arm}

\subsection{When the power supply is a forward converter followed by a linear regulator}

Uncontrolled pre-charging results in stable module capacitor voltages and power supplies to all the modules enabling switching of the IGBT switches. The voltage on each capacitor is equal to $V_{d c} / 2 n$ for ' $n$ ' number of modules per arm. However, ideal MMC operation requires initial charge on the capacitor equal to $V_{d c} / n$ [11]. Therefore it is necessary to increase the initial voltage on the capacitor to $V_{d c} / n$. As shown in the previous section, when a forward converter followed by linear regulator is used, the power supplies of all the modules are regulated and IGBTs can be switched. The capacitor voltage can therefore be increased as explained in [12]. If the capacitor voltage is to be increased to higher than $V d c / 2 n$, the modules have to operate in step-up mode. As many modules are connected in series the voltages of the module capacitors have to be balanced. The sorting algorithm [1] was used for balancing
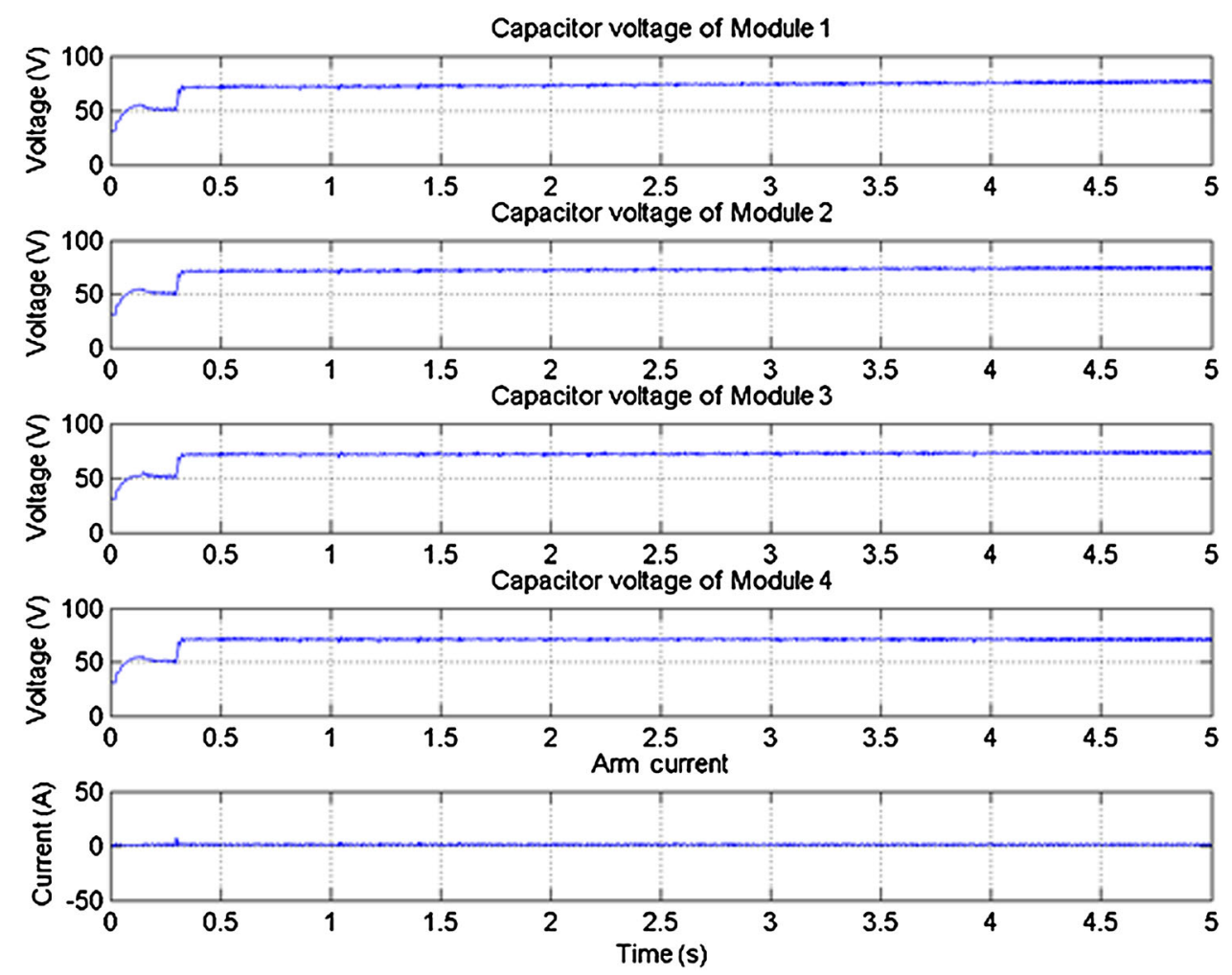

Figure 14. Uncontrolled pre-charge of capacitors when a forward converter followed by a linear regulator is used. 

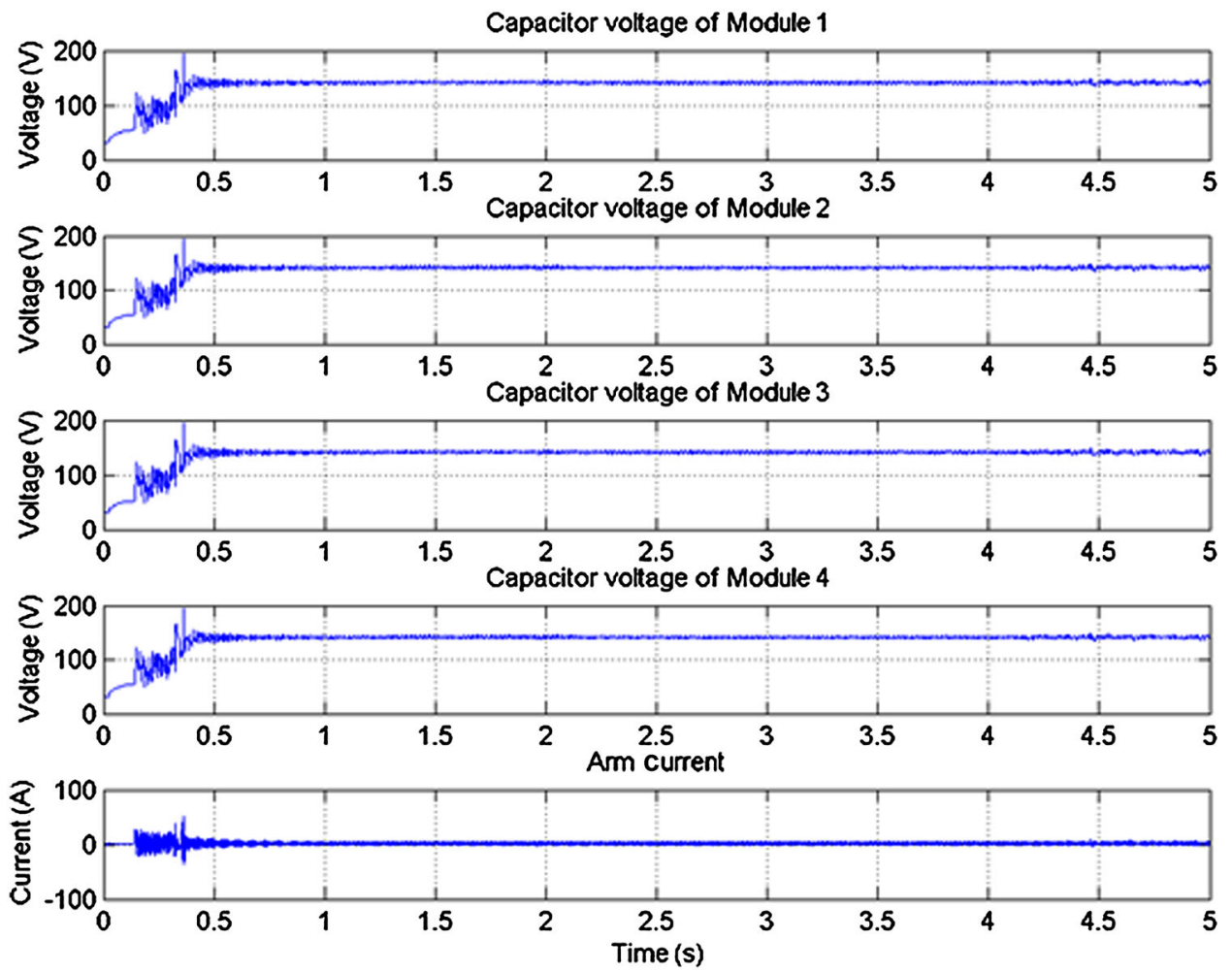

Figure 15. Controlled pre-charge of capacitors when a forward converter followed by a linear regulator is used.

capacitor voltage. For generating PWM waveforms the phase-shifted carrier PWM method [3] was used. At any instant, ' $n$ ' number of modules of one leg are on. When the arm current is in a direction charging the module capacitor, the modules with lowest $n$ voltages are inserted. When the arm current is in a direction discharging the capacitors, the $n$ modules with highest voltage are inserted, to keep the voltages of all modules balanced. All capacitors are charged and balance is maintained. Figure 15 shows controlled pre-charge of capacitors when a forward converter followed by linear regulator is used.

Figure 15 shows that the capacitors can be charged to $V_{d c} / n=150 \mathrm{~V}$ and remain stable.

\subsection{When the power supply is a regulated fly-back converter}

In this case, some of the capacitor voltages, after uncontrolled pre-charging, are not at the desired value as shown in figure 13; the corresponding power supplies will not be ready to provide the drive for the IGBT switches. On closer observation, we find that the modules, for which the power supply has reached the desired value and are ready to provide drive for IGBT switches, can be used to further charge the capacitors that do not have sufficient voltage. From figure 12 we observe that even if switches S1 or S2 cannot be switched, capacitor can still be charged if current flows through diode D1. Hence, if one of the switches S2 of any of the modules can be switched on, the current in the leg can be set so that diode D1 conducts. From figure 13, modules 1 and 2 have the minimum capacitor voltage required and their power supplies are ready to drive S1 or S2. After at least one of the power supplies is ready, if switch S2 of that module is switched 'ON', the current in the leg will be forced to increase in the direction charging the capacitors. Figure 12 shows the condition when all switches are off. When at least one of the module power supplies, say the topmost module M1 in figure 12 is ready, switch S2 of that module can be turned on. As shown in figure 16a, while the capacitor with the highest voltage gets bypassed by switch S2 and hence does not charge further, current in the leg builds up, storing energy in the series inductors, and charging all other capacitors. The switch S2 is opened and switch S1 is closed as shown in figure $16 \mathrm{~b}$, transferring the stored energy to all the capacitors that are not bypassed. The process of operating the module M1 in PWM mode continues till one more capacitor reaches a level required for getting regulated supply ready. At this stage, two of the switches S2, of modules are operated in PWM mode. The process can be continued till ' $2 n$ ' number of modules have the power supply ready. After this point, sorting algorithm as explained in the earlier part of the present section is applied till all the capacitors reach the desired voltage.

Figure 17 shows the module capacitor voltages for one arm, and the arm currents. It is noted that waveforms until 

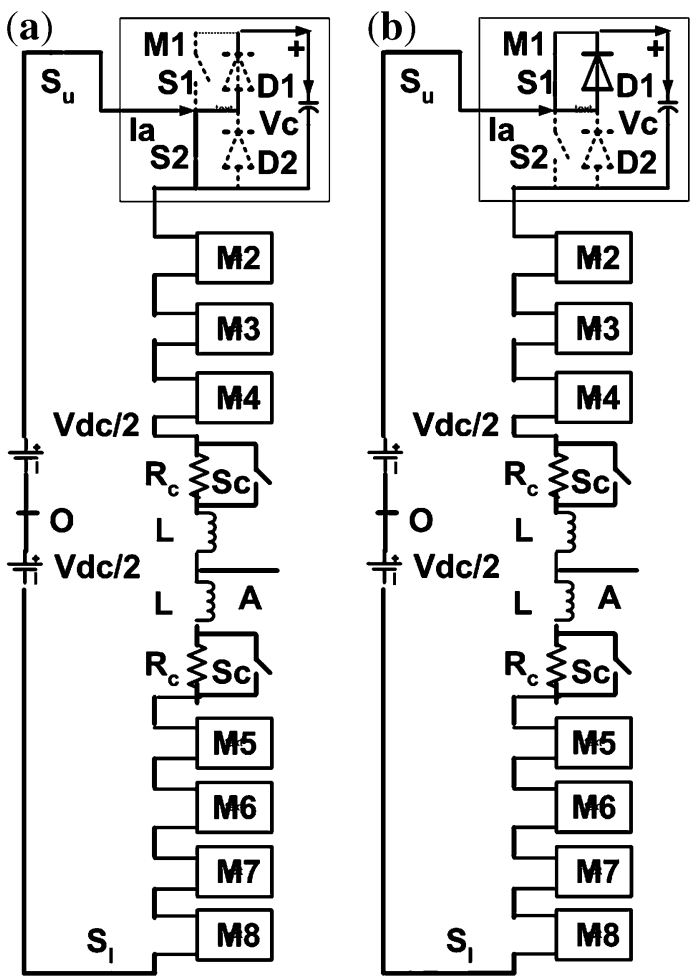

Figure 16. Controlled pre-charging of MMC when power supply is a fly-back converter: (a) module M1 bypassed and (b) module M1 inserted.
$6.4 \mathrm{~s}$ are similar to those in figure 13. At $6.4 \mathrm{~s}$, pulses to those modules that have the power supply ready, e.g., the top two modules, are released. All the module capacitors start charging and the power supplies of the modules get ready one by one. Once power supplies for ' $n$ ' number of modules in one leg are ready, sorting algorithm is started and all the capacitors get charged to the desired level. The instant of releasing the pulses is delayed here to $6.4 \mathrm{~s}$ after start, to show that even if the capacitor voltages are unstable after uncontrolled starting stage, they can be brought under control. Figure 18 shows the module capacitor voltages for one arm and the arm currents. Comparing figures 17 and 18, immediately after uncontrolled pre-charging is over, e.g., at $1 \mathrm{~s}$, all the capacitor voltages are above $55 \mathrm{~V}$ and therefore $15 \mathrm{~V}$ regulated power supply is available for all modules. Hence, if pulses to all the modules are released at this stage itself, the capacitor voltages reach the desired voltage earlier and the possibility of one or more capacitors exceeding the safe limit is avoided. Hence, it is necessary to start the controlled precharging immediately after the uncontrolled pre-charging is over.

\section{Conclusion}

An MMC module, with its control circuit powered from the module capacitor voltage, will solve the problem of EMC and high isolation requirements of transformers used in the
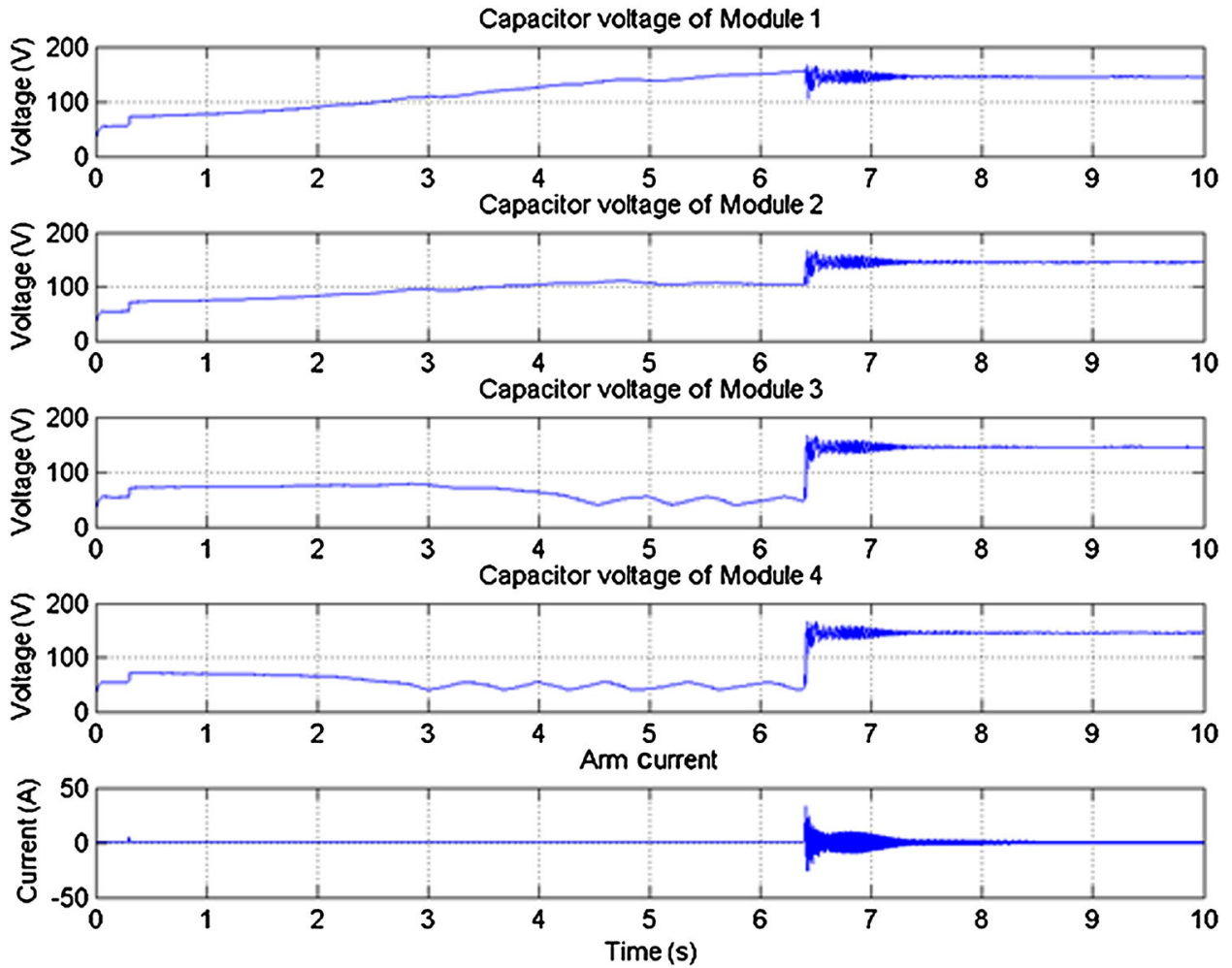

Figure 17. Delayed controlled pre-charge process of capacitors when a fly-back converter is used. 

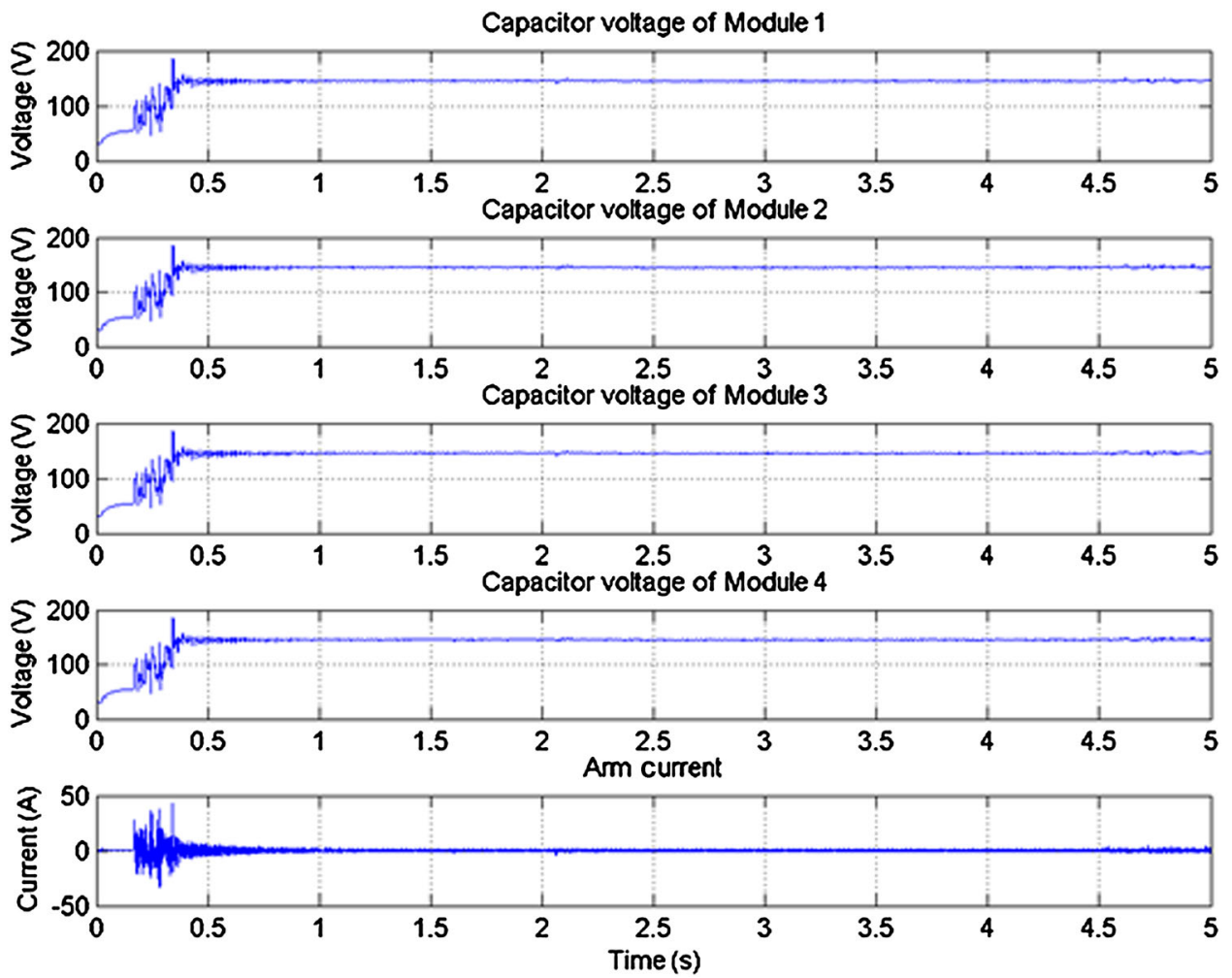

Figure 18. Controlled pre-charge process of capacitors when a fly-back converter is used.

control circuit powering part. Moreover, it reduces the total number of wires because separate wires are not required for power supplies of each module.

In MMC, the module capacitors need to be pre-charged. If pre-charged from an auxiliary supply, the disadvantage is that a separate isolated power supply is required, making the pre-charging cumbersome and expensive.

If pre-charging is done by the self-start method by charging the module capacitors from the dc bus itself, it has to be done in two stages, namely, uncontrolled pre-charging followed by controlled pre-charging.

A fly-back converter was chosen for this application as the first alternative because it is the simplest configuration with less number of components, resulting in smaller size and higher efficiency. If a regulated dc-dc converter of any other topology is used, the phenomenon will still occur because it will present a load with negative resistance characteristics to the module capacitor.

When a forward converter with fixed duty cycle in open loop, followed by a linear regulator that has the characteristics of drawing higher power at higher input voltage, is used, the module capacitor voltages are stabilized in uncontrolled pre-charging stage itself. The voltage across the module capacitor is stable as shown in figure 14. Controlled pre-charging is required to charge the capacitors to the desired voltage, to make the MMC ready to produce the desired output.
When the module power supply is derived from the flyback converter, although capacitor voltages are unstable after uncontrolled pre-charging, it is shown that the module capacitors are required to be further charged to the desired voltage. The process is explained theoretically and confirmed by simulation. However in this case it is necessary to start the controlled pre-charging of the capacitors immediately following the uncontrolled pre-charging to avoid module capacitors over-voltages, beyond the safe range.

\section{Acknowledgements}

The authors would like to thank the NaMPET (National Mission on Power Electronics Technology launched by the Department of Electronics and Information Technology under Ministry of Communications and Information Technology, Government of India), which supports the present work.

\section{Appendix}

Referring to Eq. (6), all the co-efficients in the numerator and the denominator of the transfer function are positive. The denominator is a third-order equation. Consider a thirdorder polynomial given by the equation 


$$
a_{0} s^{3}+a_{1} s^{2}+a_{2} s+a_{3}=0 .
$$

Applying Routh's Stability Criterion to confirm that the system is stable, the conditions for all roots to have negative real part are given by

$$
a_{1} a_{2}>a_{0} a_{3} .
$$

Comparing Eq. (11) with the denominator of Eq. (6) results in the following:

$$
\begin{gathered}
a_{0}=R_{1} R_{2} L C^{2}, \\
a_{1}=\left(R R_{1} R_{2} C^{2}+L C R_{1}+L C R_{2}\right), \\
a_{2}=\left(L+C R R_{1}+C R R_{2}\right), \\
a_{3}=\left(R+R_{1}+R_{2}\right) .
\end{gathered}
$$

To evaluate the parameters in these equations, values of $R_{1}$ and $R_{2}$, which represent incremental load resistances as seen by the module capacitors, have to be found out. Referring to figure 10 , the module capacitor, voltage $V_{c}$ is reduced to a voltage $V_{i}$ by the forward converter operating at a fixed duty cycle by a factor of 5 . The input resistance offered by the forward converter is the effective load resistance seen by the module capacitor. If the incremental quantities are indicated by small letters, the load resistance $R_{1}$ is given by the relation

$$
R_{1}=\frac{v_{c}}{i_{c}}
$$

Assuming the losses in the forward converter to be negligible, the current $i_{c}$ and the voltage $v_{c}$ are given by

$$
\begin{gathered}
i_{c}=0.2 i_{i}, \\
v_{c}=5 v_{i} .
\end{gathered}
$$

The linear regulator is a series pass regulator and its output current $i_{0}$ is the same as the input current $i_{i}$. The output voltage $V_{0}$ is about $15 \mathrm{~V}$ and average current drawn from the output is $0.15 \mathrm{~A}$. Hence the effective resistance at the output of the linear regulator is $100 \Omega$. If $v_{0}$ is the incremental voltage change in the output voltage $V_{0}$ and $v_{i}$ is the input, the regulator, from the data for input voltage regulation for TL783 of

$$
\begin{gathered}
\frac{v_{o}}{v_{i}}=0.004 \frac{V_{o}}{100}=0.0006, \\
i_{c}=0.2 i_{0}=0.2 \frac{V_{o}}{100}=0.002 v_{0} .
\end{gathered}
$$

From Eq. (17)-(21) the resistance $R_{1}$ is given

$$
R_{1}=4.17 \mathrm{M} \Omega \text {. }
$$

Hence the incremental load resistance $R_{1}$ for the module capacitor, as shown by (22), is $4.17 \mathrm{M} \Omega$. With identical
MMC modules the value of the second module load resistance $R_{2}$ is assumed to be the same.

With these values of $R_{1}$ and $R_{2}, L$ equal to $3 \mathrm{mH}$ and $C$ equal to $1 \mathrm{mF}$, Eqs. (13)-(16) are evaluated. The necessary and sufficient condition for the system is given by (12). Eq. (12) is satisfied as $a_{1} a_{2}=3600 \times 10^{9}$ and $a_{0} a_{3}=$ $431 \times 10^{9}$. Hence it is proved that the system is stable.

\section{References}

[1] Lesnicar A and Marquardt R 2003 An innovative modular multilevel converter topology suitable for a wide power range. In: Proceeding of the IEEE Bologna Power Tech Conference, Italy

[2] Hagiwara M, Maeda R and Akagi H 2011 Control and analysis of the modular multilevel cascade converter based on double-star chopper-cells (MMCC-DSCC). IEEE Trans. Power Electron. 26:1649-1658

[3] Konstantinou G S and Agelidis V G 2009 Performance evaluation of half-bridge cascaded multilevel converters operated with multicarrier sinusoidal PWM techniques. In: Proceeding of the IEEE Conference on Industrial Electronics and Applications, Xi'an

[4] Siemaszko D, Antonopoulos A, Ilves K, Vasiladiotis M, Ngquist L and Hans-Peter Nee 2010 Evaluation of control and modulation methods for modular multilevel converters. In: Proceeding of the International Power Electronics Conference, Sapporo

[5] Tu Q, Xu Z and Zhang J 2010 Circulating current suppressing controller in modular multilevel converter. In: Proceedings of the 36th Annual Conference of the IEEE Industrial Electronics Society, Glendale

[6] Hagiwara M and Akagi H 2009 Control and experiment of pulse width modulated modular multilevel converters. IEEE Trans. Power Electron. 24:1737-1746

[7] Marquardt R and Lesnicar A 2004 New concept for high voltage modular multilevel converter. In: Proceeding of the Power Electronics Specialists Conference and Exhibition, Aachen, Germany

[8] Glinka M 2004 Prototype of multiphase modular-multilevelconverter with 2-MW power rating and 17-level-output-voltage. In: Proceeding of the Power Electronics Specialists Conference

[9] Joshi S, Sreejith M R, Chandorkar M C and Shukla A 2014 MMC modules with control circuit powered from module capacitor voltage. In: Proceedings of the IEEE PEDES Conference Mumbai, India

[10] Sreejith M R 2014 Electrical source emulation using modular multilevel converter. M.Tech. Thesis. Mumbai, India: Deptartment of Electrical Engineering, IIT Bombay

[11] Li B, Xu D, Zhang Y, Yang R and Wang G 2015 Closedloop pre-charge Control of modular multilevel converters during start-up processes. IEEE Trans. Power Electron. 30:(2) 524-531

[12] Shi K, Shen F, Lv D, Lin P, Chen M and Xu D 2012 A novel start-up scheme for modular multilevel converter. In: Proceedings of the IEEE Energy Conversion Congress and Exposition 